\title{
Isoproterenol increases histone deacetylase 6 expression and cell migration by inhibiting ERK signaling via PKA and Epac pathways in human lung cancer cells
}

\author{
Jeong Ah Lim and Yong-Sung Juhnn
}

\begin{abstract}
Stress conditions are correlated with tumor growth, progression and metastasis. We hypothesized that stress signals might affect tumor progression via epigenetic control of gene expression and investigated the effects of stress signals on the expression levels of histone deacetylases (HDACs) and the underlying mechanisms of these effects in lung cancer cells. Treatment with isoproterenol (ISO), an analog of the stress signal epinephrine, increased the expression of HDAC6 protein and mRNA in H1299 lung cancer cells. ISO caused the deacetylation of $\alpha$-tubulin and stimulated cell migration in an HDAC6-dependent manner. HDAC6 expression was increased by treatment with selective activators of cAMP-dependent protein kinase (PKA) or exchange protein activated by CAMP (Epac). ISO activated Rap1 via Epac, and constitutively active Rap1A increased the HDAC6 level; however, the knockdown of Rap1A decreased the 8-(4-cholorophenylthio)-2'-0-methyl-cAMP-induced increase in HDAC6 expression. Both PKA and Rap1A decreased c-Raf activation to inhibit extracellular signal-regulated kinase (ERK) signaling. Inhibition of ERK caused an increase in HDAC6 expression, and constitutively active MEK1 decreased the ISO-induced HDAC6 expression. We concluded that ISO increases HDAC6 expression via a PKA/Epac/ERK-dependent pathway that stimulates the migration of lung cancer cells. This study suggests that stress signals can stimulate the migration of cancer cells by inducing HDAC6 expression in lung cancer cells.
\end{abstract}

Experimental \& Molecular Medicine (2016) 48, e204; doi:10.1038/emm.2015.98; published online 15 January 2016

\section{INTRODUCTION}

The stress response is an innate and adaptive response to stressors and restores the non-stressed homeostatic state. The stress response elicits specific cognitive, behavioral and physiological phenomena and results from the translation of psychosocial stimuli into peripheral biological signals by the central nervous system. For example, a variety of stressors cause alterations in various neuroendocrine hormones, particularly catecholamines and cortisol, via the activation of the sympathetic nervous system and the hypothalamicpituitary-adrenal axis. ${ }^{1}$

Stress conditions affect many pathological outcomes of various diseases, including cancer. Tumor growth, progression and metastasis have been revealed to correlate with stress responses in clinical and epidemiological studies. ${ }^{2,3}$ Stress hormones, such as epinephrine and norepinephrine, regulate the production of cytokines, growth factors and matrix metalloproteinases to promote tumor growth, progression and metastasis in many caners. ${ }^{4}$ Thus the understanding of the mechanisms by which stress responses affect cancer growth and progression may contribute to improvements in cancer management. $^{5}$

Carcinogenesis and tumor progression involve alterations of the genetic and epigenetic regulations of the expression levels of multiple genes. Changes in lysine acetylation on the histone tail are among the major epigenetic regulatory mechanisms that are found in many cancers. ${ }^{6}$ The acetylation statuses of histone proteins are balanced by two counteracting types of enzyme: histone acetyltransferases and histone deacetylases (HDACs). ${ }^{7}$ The expression of HDACs is higher in tumor tissues than the surrounding tissues, ${ }^{8}$ and the overexpression of HDACs is associated with crucial events in tumorigenesis, such as the epigenetic repression of tumor-suppressor genes and genes that encode DNA damage repair enzymes. 9 Thus HDACs are

Department of Biochemistry and Molecular Biology and Cancer Research Institute, Seoul National University College of Medicine, Seoul, Korea Correspondence: Professor Y-S Juhnn, Department of Biochemistry and Molecular Biology and Cancer Research Institute, Seoul National University College of Medicine, 103 Daehak-ro, Jongno-gu, Seoul 110-799, Korea.

E-mail: juhnn@snu.ac.kr

Received 14 September 2015; revised 5 October 2015; accepted 6 October 2015 
implicated in tumorigenesis and progression, and targeting HDACs is a promising potential therapeutic strategy. HDAC inhibitors have emerged as a new class of anticancer drugs based on their effects on the proliferation and apoptosis of cancer cells. ${ }^{10}$

We hypothesized that stress signals might affect tumor growth, progression and metastasis via the epigenetic control of gene expression. HDAC1 has been reported to be involved in the regulation of kappa-opioid receptor expression by behavioral stress. ${ }^{11}$ Chronic social stress is associated with decreased levels of HDAC2 in the mouse brain. ${ }^{12}$ However, the effects of stress on the expression levels of HDACs in cancer cells are not clearly understood; thus we aimed to investigate the effects of stress signals on the expression levels of HDACs and their underlying mechanisms in lung cancer cells. We found that isoproterenol (ISO), an analog of the stress hormone epinephrine, increases HDAC6 expression via cAMP-dependent protein kinase (PKA)/exchange protein activated by cAMP (Epac)-Raf-mitogen-activated extracellular signal-regulated kinase (MEK)-extracellular signal-regulated kinase (ERK) pathway that deacetylates $\alpha$-tubulin to promote migration of lung cancer cells.

\section{MATERIALS AND METHODS}

\section{Cell culture and reagents}

The human non-small cell lung cancer H1299 and A549 cells were purchased from the Korea Cell Line Bank (Seoul, Korea). These cells were grown in Dulbecco's Modified Eagle's Medium (DMEM) containing 10\% fetal bovine serum (FBS) (Welgene, Taegu, Korea) and 100 units $\mathrm{ml}^{-1}$ penicillin/streptomycin. The cells were cultured in a $5 \% \mathrm{CO}_{2}$ incubator at $37^{\circ} \mathrm{C}$.

Forskolin and SP600125 were purchased from Calbiochem (San Diego, CA, USA). SB203580 was purchased from Cayman (Ann Arbor, MI, USA). Actinomycin D, dimethyl sulfoxide, Dulbecco's phosphate-buffered saline, H89, ISO, PD98056, PD0325901 and prostaglandin $\mathrm{E}_{2}\left(\mathrm{PGE}_{2}\right)$ were purchased from Sigma Chemicals (St Louis, MO, USA). N6-phenyl-cAMP (6-Phe-cAMP) and 8-(4-cholorophenylthio)-2'-O-methyl-cAMP (8-pCPT-cAMP) were purchased from the Biolog Life Science Institute (Bremen, Germany).

\section{Expression plasmids and transient transfection}

The expression plasmid of the EE-tagged constitutively active mutant of Gas (GasQ227L) was purchased from the Missouri S\&T cDNA Resource Center (Rolla, MO, USA). The GosQ277L mutant has a substitution of Leu-277 for Gln-277 that results in the inactivation of the intrinsic GTPase, which causes constitutive activation of the protein. Wild-type and dominant-negative cAMP response element-binding protein (CREBs; S133A, R287L) were gifts from Dr Sahng-June Kwak (Dankook University, Cheonan, Korea). CaRap1A/1B was kindly provided by Keith Burridge (University of North Carolina, Chapel Hill, NC, USA), ${ }^{13}$ the dominant-negative PKA in MT-REVab was provided by Dr G Stanley McKnight (University of Washington, Seattle, WA, USA), and the catalytic subunit of PKA was provided by Dr SH Green. ${ }^{14}$ The short hairpin RNAs (shRNAs) against HDAC6 and Rap1 and the control shRNA were purchased from Sigma Chemicals. Small interfering RNAs (siRNAs) against Epac1 and Epac2 were from Santa Cruz Biotechnology (Santa Cruz, CA, USA). Decoy oligonucleotides for the CRE (CRE decoy) were prepared as described previously. ${ }^{15}$ The H1299 cells were transfected with expression constructs and siRNAs using Lipofectamine 2000 (Invitrogen, Carlsbad, CA, USA).

\section{Quantitative reverse transcription PCR (qPCR)}

The total RNA was isolated from the cells using the TRIzol reagent (Invitrogen). First-strand complementary DNA (cDNA) was synthesized using oligo-dT primers and the SuperScript II Reverse Transcriptase (Invitrogen). The specific oligonucleotide primers used in the qPCR were as follows: for HDAC6, 5'-TCAGGTCTACTG TGGTCGTT-3' and 5'-TCTTCACATCTAGGAGAGCC-3'; and for glyceraldehyde-3-phosphase dehydrogenase (GAPDH), 5'-ACCACA

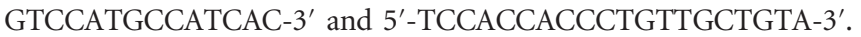

The qPCR was performed in a 20- $\mu \mathrm{l}$ mixture composed of forward and reverse primers $(5 \mathrm{pmol})$ and SYBR Premix Ex Taq (Takara Bio Inc., Japan) using a C1000 thermal cycler (Bio-Rad, Hercules, CA, USA). After 40 cycles of PCR, the average threshold cycle (Ct) values from triplicate $\mathrm{qPCR}$ experiments were normalized against the average $\mathrm{Ct}$ value of GAPDH.

\section{Western blotting analysis}

The expression levels of the proteins were analyzed by western blotting using specific antibodies. Antibodies against HDAC6 and Epac were purchased from Santa Cruz Biotechnology. Antibodies against HDAC4, acetylated tubulin, tubulin and $\beta$-actin were from Sigma Chemicals. Antibodies against HDAC7, CREB, phospho-CREB (Ser-133), c-Raf, phospho-c-Raf at Ser-338 and Ser-259, MEK1/2, phospho-MEK1/2, p44/42 mitogen-activated protein kinase (MAPK), phospho-p44/42 MAPK and Rap1A/1B were purchased from Cell Signaling Technology (Danvers, MA, USA). The HDAC9 antibody was from Abcam (Cambridge, UK). The HDAC5 antibody was from Alexis Biochemicals (Plymouth Meeting, PA, USA). The horseradish peroxidase-conjugated goat anti-mouse and anti-rabbit IgG antibodies were purchased from Zymed (South San Francisco, CA, USA) or Santa Cruz Biotechnology. The blots were incubated with an enhanced chemiluminescence substrate mixture (Pierce, Chester, UK), and the resulting blot images were then recorded with an LAS-3000 luminescent image analyzer system (Fuji, Tokyo, Japan). The densities of the protein bands were quantified using the Multi Gauge v3.0 software (Fuji), and the protein amounts are expressed as multiples of the corresponding densities of the control.

\section{Transwell migration assay}

H1299 cells were transfected with shRNA targeting HDAC6 or scrambled sequences and incubated for $48 \mathrm{~h}$. The cells (10000) were then seeded onto the upper chamber $(8-\mu \mathrm{m}$ pore size) of a 24 -well plate (Corning, Lowell, MA, USA) containing DMEM, 1\% FBS and $20 \mu \mathrm{M}$ ISO. The lower chamber was filled with DMEM containing 10\% FBS and $20 \mu \mathrm{m}$ ISO. After incubation for $16 \mathrm{~h}$, the migrated cells were fixed and stained using Diff-Quik solution (Sysmex, Kobe, Japan), and the non-migrated cells were removed with a cotton swab. The cell numbers were counted in five different microscopic fields (Leica DFL 290, Wetzlar, Germany).

\section{Assay of Rap1 activity}

Rapl activity was assayed by analyzing the binding of the activated proteins to the Rap1-binding domain (RBD) of the ral guanine nucleotide exchange factor (RalGDS) protein. The plasmid encoding a GST fusion protein containing the 97 amino-acid RBD of the RalGDS protein (pGEX RalGDS-RA) was a gift from JH Chung 
(NIH, Bethesda, MD, USA). ${ }^{16}$ The cell lysates were incubated with GST-RalGDS RBD protein that was preincubated with glutathioneSepharose $4 \mathrm{~B}$ (GE Healthcare, NJ, USA) at $4{ }^{\circ} \mathrm{C}$ with agitation. The beads were washed three times with lysis buffer and then subjected to immunoblotting analysis.

\section{Data analysis}

All experiments were independently repeated at least three times, and the data are presented as the means \pm s.e. The non-parametric Mann-Whitney $U$-test was used to analyze the mean values, and $P$-values $<0.05$ were considered statistically significant.

\section{RESULTS}

ISO signaling increases the expression levels of HDAC6 protein and mRNA in H1299 lung cancer cells

To examine the effects of stress signals on the expression levels of HDACs, we treated H1299 human lung cancer cells with ISO, which is an analog of the epinephrine stress hormone, and the expression levels of HDACII family proteins were analyzed by western blotting. Treatment with ISO increased the expression of HDAC6 without significantly altering the expression levels of the other tested HDACII proteins $(P<0.05$; Figure 1a). Because ISO binds to and activates $\beta$-adrenergic receptors, which in turn activate stimulatory GTP-binding protein (Gs), we examined the effect of Gs on the expression levels of the HDACII family proteins. Transient expression of constitutively active GosQL also increased the expression of HDAC6 protein alone (Supplementary Figure S1). Furthermore, treatment of the H1299 cells with another Gas-coupled receptor agonist $\left(\mathrm{PGE}_{2}\right)$ or an adenylyl cyclase activator (forskolin) also increased HDAC6 expression (Figure 1b). When the cells were treated with ISO, the expression of HDAC6 mRNA increased at $6 \mathrm{~h}$ and reached a plateau at $30 \mathrm{~h}$, and the expression of HDAC6 protein began to increase at $12 \mathrm{~h}$ (Figure 1c). To examine whether increased transcription might have caused the increase in HDAC6 expression, the effect of transcription inhibition via actinomycin D treatment on the ISO-induced HDAC6 expression was assessed. Treatment with actinomycin D abolished the ISO-induced increases in HDAC6 mRNA and protein expression levels (Figure 1d). These results indicate that ISO signaling increases HDAC6 protein expression by stimulating the transcription of the HDAC6 gene via cAMP signaling in lung cancer cells.

\section{ISO decreases the acetylation of $\alpha$-tubulin and increases the migration of H1299 lung cancer cells in an HDAC6-dependent manner}

HDAC6 deacetylates many non-histone proteins, including $\alpha$-tubulin, and the acetylation of $\alpha$-tubulin is involved in the regulation of cell migration. Thus the effects of ISO on the acetylation of $\alpha$-tubulin and cell migration were examined. Treatment with ISO significantly reduced the acetylation of $\alpha$-tubulin at 36 and $48 \mathrm{~h}$ after treatment (Figure 2a). When HDAC6 expression was knocked down via transfection with HDAC6 shRNA, the acetylation of $\alpha$-tubulin increased
(Figure 2b). Treatment with ISO increased the migration of the H1299 cells in the transwell migration assay, and this increase in migration was blocked by knockdown of HDAC6 with a specific shRNA (Figure 2c). These results indicate that ISO decreases the acetylation of $\alpha$-tubulin and promotes cell migration by increasing HDAC6 expression in H1299 lung cancer cells.

\section{ISO increases HDAC6 by inhibiting c-Raf-MEK-ERK pathways}

To probe the signaling pathway that mediated the HDAC6increasing effect of ISO signaling, the effects of MAPKs on HDAC6 expression were assessed following treatment with MAPK-specific inhibitors. Treatment with the ERK inhibitor (PD98059) caused an increase in HDAC6 expression that was similar to that of ISO treatment. However, treatments with a c-Jun N-terminal kinase inhibitor (SP600215) and a p38 inhibitor (SB203580) did not significantly alter HDAC6 expression levels (Figure 3a). Treatment with PD0325901, another ERK inhibitor, also caused a significant increase in HDAC6 expression (Supplementary Figure S2). Treatment with PD98059 increased the HDAC6 mRNA level, and the inhibition of transcription via actinomycin D abolished the effects of PD98059 on HDAC6 mRNA and protein (Figure 3b). Moreover, treatment with ISO decreased the activating phosphorylation of ERK1/2, the upstream MEK1/2 and c-Raf (S338) from $30 \mathrm{~min}$ until $4 \mathrm{~h}$ after treatment (Figure $3 \mathrm{c}$ ). Furthermore, the expression of constitutively active MEK1 (caMEK1) abolished the ISO-induced HDAC6 expression (Figure 3d). Additionally, treatment of A549 cells, that is, another human lung cancer cell line, with forskolin also inhibited the c-Raf-MEK1/2-ERK1/2 activity and increased HDAC6 expression (Supplementary Figure S3). These results indicate that ISO increased HDAC6 expression via inhibition of the c-Raf-MEK1/2-ERK1/2 signaling pathway in lung cancer cells.

\section{Both the PKA and Epac pathways mediate ISO-induced inhibitions of c-Raf}

To investigate the mechanism by which ISO inhibited c-Raf-MEK1/2-ERK1/2 to increase HDAC6 expression, we analyzed the roles of the PKA and Epac pathways, which are the two major signaling pathways that are activated by CAMP. The expression of a dominant-negative PKA did not block the ISO-induced HDAC6 expression (Figure 4a). Similarly, knockdown of Epac1/2 with specific siRNAs did not block the ISO-induced HDAC6 expression (Figure 4b). However, the simultaneous transfection of dominant-negative PKA and Epac1/2 siRNAs abolished the ISO-induced HDAC6 expression (Figure 4c). The involvements of PKA and Epacs in HDAC6 expression were confirmed in a study utilizing selective agonists. Treatment with the PKA-selective agonist N6-phenyl-cAMP (6-Phe-cAMP) or the Epac selective agonist 8-pCPT-2'-O-Me-cAMP (8-pCPT-cAMP) decreased the activating phosphorylation of c-Raf at Ser-338 and increased the inhibitory phosphorylation of c-Raf at Ser-259. The inhibitions 

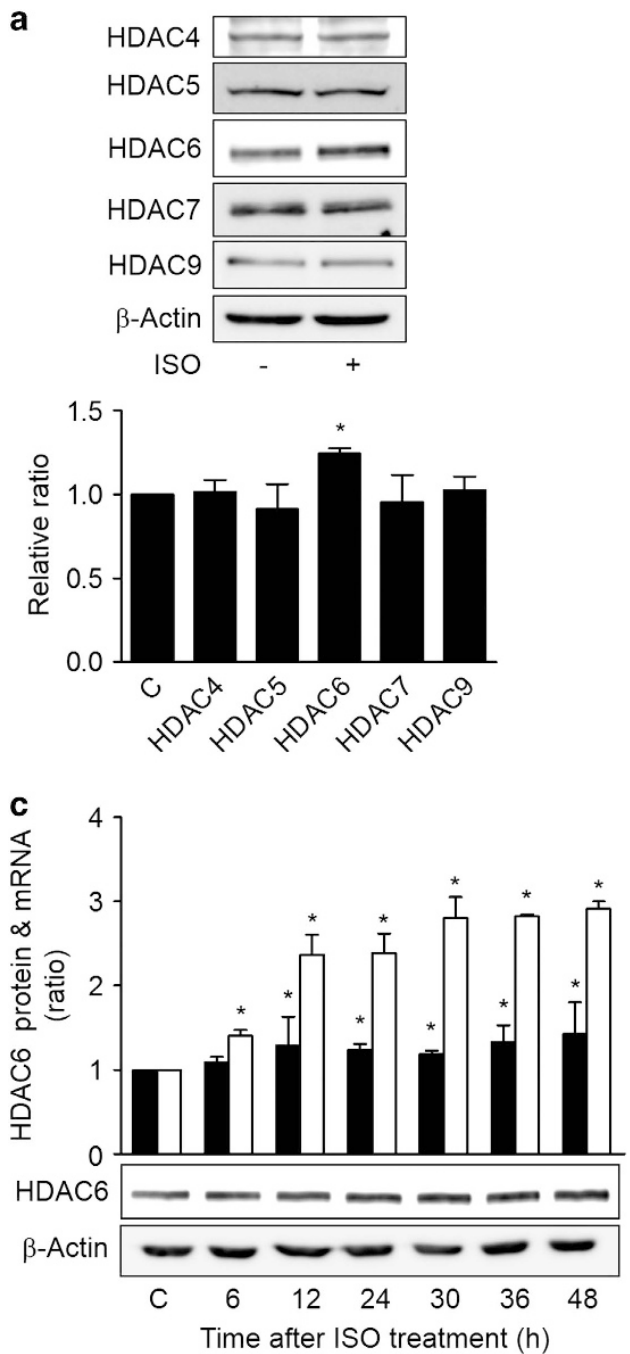

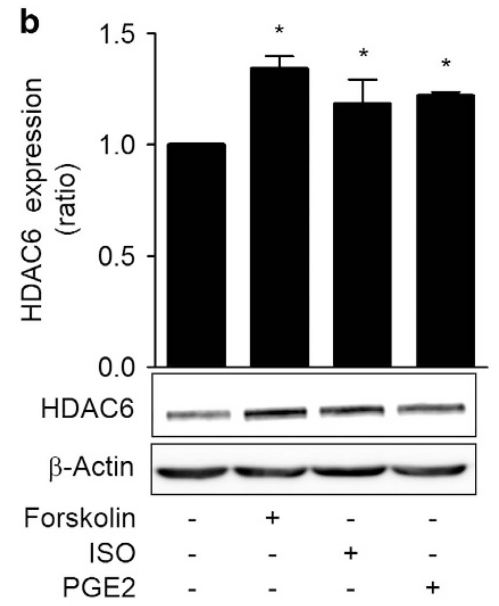

d

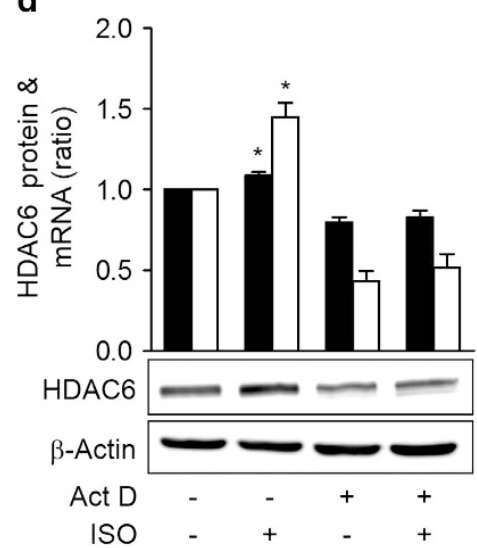

Figure 1 Isoproterenol signaling increases HDAC6 expression in lung cancer cells. (a) Effects of isoproterenol on the expression levels of type II HDACs in H1299 human lung cancer cells. (b) Effects of cAMP signaling on the expression of HDAC6. (c) Temporal patterns of the expression levels of HDAC6 protein and mRNA following isoproterenol treatment. (d) Effects of transcription inhibition on the isoproterenolinduced increase in HDAC6 expression. $\mathrm{H} 1299$ cells were pretreated with actinomycin D $(8 \mu \mathrm{m})$ for $2 \mathrm{~h}$ before isoproterenol treatment $(20 \mu \mathrm{M})$, and the cells were harvested at $30 \mathrm{~h}$ for analyses of the mRNA levels and at $48 \mathrm{~h}$ for the western blotting analysis. $\mathrm{H} 1299 \mathrm{human}$ lung cancer cells were treated with $20 \mu \mathrm{m}$ isoproterenol (ISO), $20 \mu \mathrm{m}$ forskolin or $20 \mu \mathrm{M} \mathrm{PGE}_{2}$ and harvested at $48 \mathrm{~h}$ or at the indicated time points for western blotting analysis. $\beta$-Actin was analyzed as a loading control. The western blotting band densities were quantified using the Multi Gauge v.2.3 software and are expressed as the ratio relative to the control band density. The HDAC6 mRNA level was analyzed by quantitative real-time PCR and normalized to GAPDH mRNA and is expressed as the ratio relative to the control level. The filled bar represents the HDAC6 protein, and the empty bar represents the HDAC6 mRNA. Asterisks $\left({ }^{*}\right)$ indicate statistically significant differences compared with the respective control $(P<0.05$, Mann-Whitney $U$-test).

of c-Raf by these agonists resulted in decreased phosphorylation of the downstream MEK1/2 and ERK1/2, which caused an increase in HDAC6 expression similar to that of ISO treatment (Figure 4d). Moreover, the expression of the catalytic subunit of PKA also induced HDAC6 expression (Figure 4e). Additionally, the mediation of the 6-Phe-cAMP effect on HDAC6 expression by PKA was confirmed by the abolishment of the 6-Phe-cAMP effect by the expression of a dominantnegative PKA (Supplementary Figure S4). These results indicate that both the PKA and Epac pathways mediated the ISOinduced inhibition of c-Raf and increase in HDAC6 expression.
Rap1A mediates the Epac-induced inhibition of c-Raf To study how the Epac pathway inhibits c-Raf, we assessed the role of Rap1, which is a well-known downstream target of Epac. The effect of the Epac selective activator, 8-pCPT-cAMP, on HDAC6 expression was blocked by the knockdown of Epac with siRNA, which confirmed the selectivity of the agonist for Epac (Figure 5a). Treatment with 8-pCPT-cAMP or ISO increased the amount of Rap1 bound to RBD of the RalGDS protein in a pull-down assay (Figure 5b). Because Rap1 has two isoforms, that is, Rap1A and Rap1B, we determined which isoform mediated the HDAC6-expressing effect by transfecting 

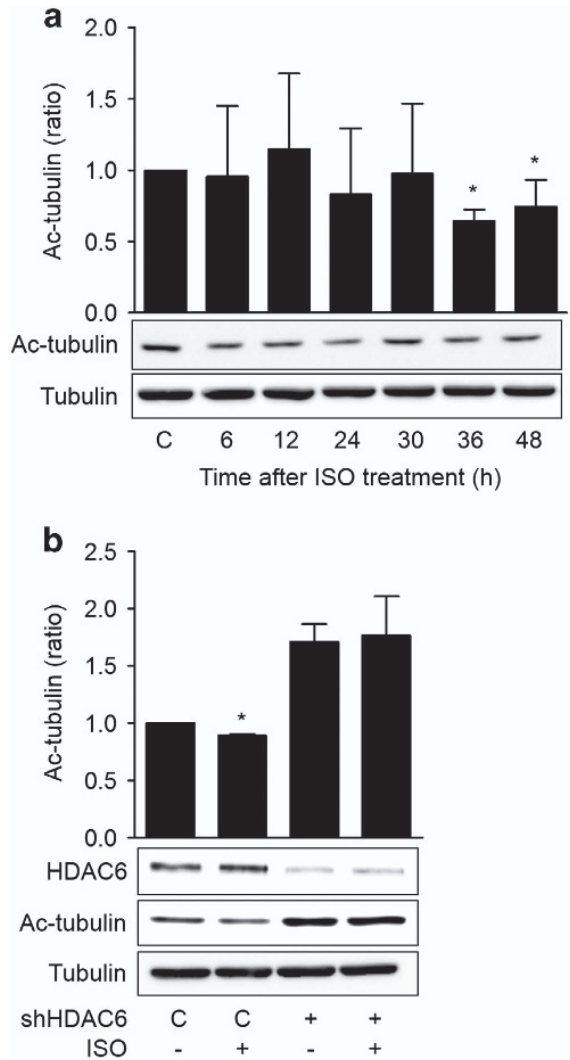
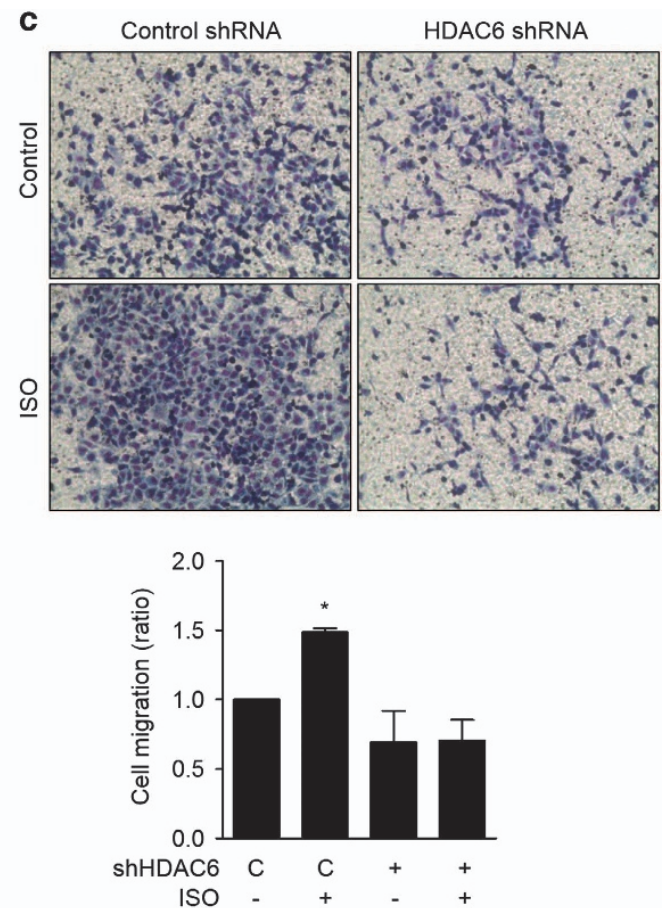

Figure 2 Isoproterenol increases the migration of H1299 lung cancer cells via the HDAC6-dependent deacetylation of $\alpha$-tubulin. (a) Effects of isoproterenol on the acetylation of $\alpha$-tubulin. H1299 cells were treated with $20 \mu \mathrm{m}$ isoproterenol (ISO), and the levels of acetylated $\alpha$-tubulin (Ac-tubulin) and total $\alpha$-tubulin at the indicated time points were assessed by western blotting. (b) Effects of HDAC6 knockdown on the isoproterenol-induced deacetylation of $\alpha$-tubulin. H1299 cells in 100-mm dishes were transfected with shRNA targeting HDAC6 or control shRNA $(10 \mu \mathrm{g})$ and incubated for $24 \mathrm{~h}$. Then the cells were treated with $20 \mu \mathrm{m}$ isoproterenol for an additional $48 \mathrm{~h}$ prior to harvesting for western blotting analysis. (c) Effects of isoproterenol on the migration of H1299 lung cancer cells. A transwell migration assay was performed. In brief, H1299 cells were transfected with shRNA targeting HDAC6 or control shRNA, and the cells were seeded onto the upper chamber containing $20 \mu \mathrm{m}$ isoproterenol. After $16 \mathrm{~h}$, the migrated cells were fixed and stained, and representative photographs ( $\times 100$ magnification) of the migrated cells are presented. The migrated cells were counted in five different microscopic fields, and the averages were calculated. The values presented are the means \pm s.ds. of three independent experiments. Asterisks $\left({ }^{*}\right)$ indicate significant differences from the isoproterenol-untreated control $(P<0.05$, Mann-Whitney $U$-test).

each of the constitutively active forms of Rap1 into H1299 cells. H1299 cells that expressed the constitutively active Rap1A (caRap1A) exhibited increased HDAC6 expression and decreased phosphorylations of c-Raf, MEK1/2 and ERK1/2, but the cells that expressed the constitutively active Rap $1 \mathrm{~B}$ (caRap1B) did not exhibit increased HDAC6 expression or decreased phosphorylation of c-Raf (Figure 5c). The knockdown of Rap1A with shRNA abolished the 8-pCPT-cAMPinduced HDAC6 expression, which confirmed the mediating role of Rap1A (Figure 5d). These results indicate that Epac increased HDAC6 expression by activating Rap1A.

To study whether ERK mediates ISO-induced HDAC6 expression via CREB, a well-known transcription factor that is activated by PKA, the effect of CREB inhibition by transfecting dominant-negative CREBs or CRE decoy oligonucleotides on PD98059-induced expression of HDAC6 were analyzed. The PD98059-induced increase in HDAC6 expression was not abolished by transfection of dominant-negative CREBs (S133A and R287L) or CRE decoy oligonucleotides
(Figures $6 \mathrm{a}$ and $\mathrm{b}$ ). CREB inhibition by the dominant-negative CREB (S133A) or CRE decoy oligonucleotides also abolished the increase in HDAC6 expression by ISO treatment (Figure 6c). These results suggest that ISO increases HDAC6 expression in a CREB-independent pathway.

\section{DISCUSSION}

This study investigated the effects of stress signals on HDAC expression and their underlying mechanisms in human lung cancer cells. We found that ISO signaling increases HDAC6 expression by inducing transcription through a PKA/Epac/ ERK-dependent pathway and that the ISO-induced increase in HDAC6 expression stimulates the migration of H1299 lung cancer cells (Figure 6d).

Our finding that ISO signaling increased the expression of HDAC6, which stimulated the migration of lung cancer cells, is supported by the result that treatment with ISO increased the expression levels of HDAC6 mRNA and protein without affecting the expression levels of other class II HDACs in 


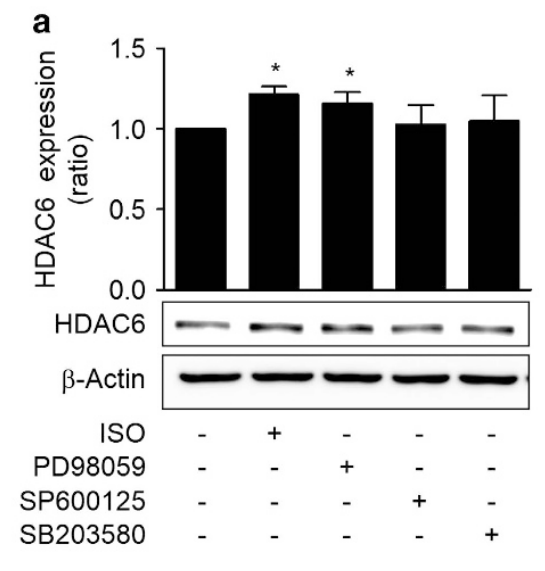

C

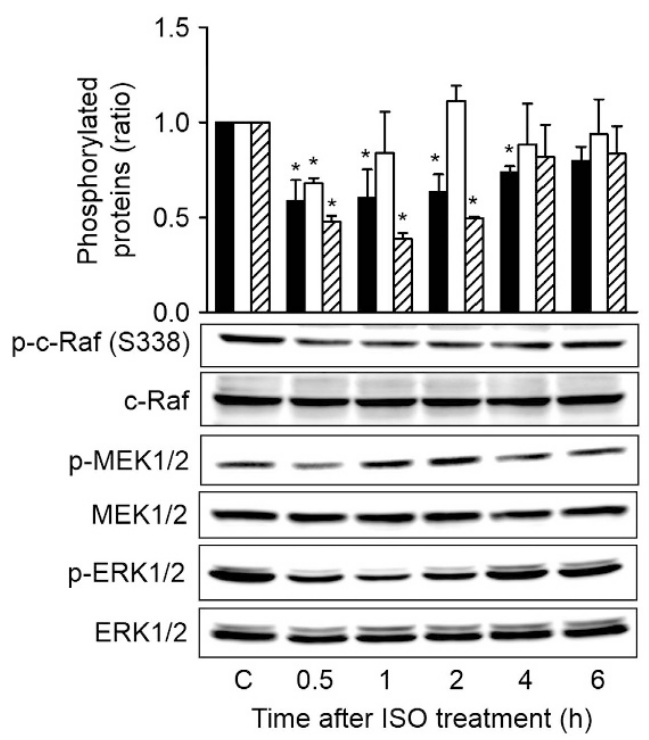

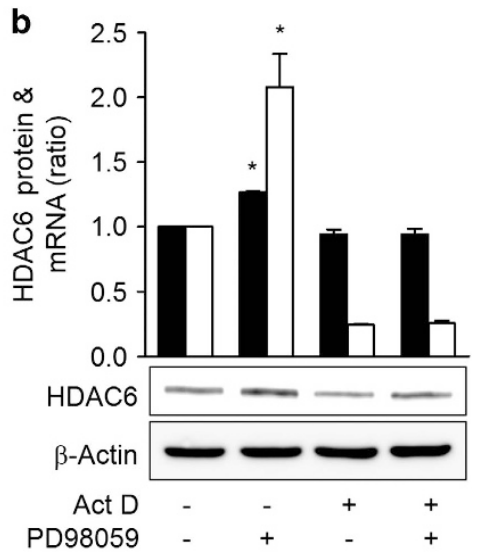

d

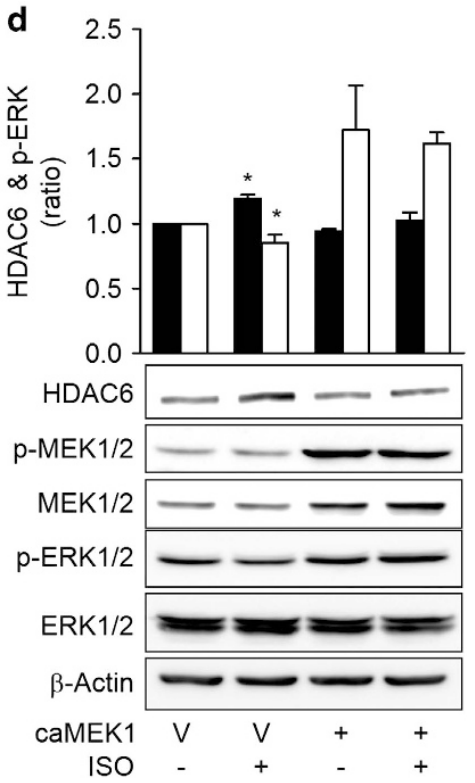

Figure 3 Isoproterenol increases HDAC6 by inhibiting c-Raf-MEK-ERK pathways. (a) Effects of MAPK inhibition on HDAC6 expression. H1299 cells were treated with $20 \mu \mathrm{m}$ isoproterenol (ISO), $20 \mu \mathrm{m}$ PD98059 (PD), $10 \mu \mathrm{m}$ SP600215 (SP) or $20 \mu \mathrm{m} \mathrm{SB03580} \mathrm{(SB)} \mathrm{for} 48 \mathrm{~h}$, and HDAC6 expression was then analyzed by western blotting. (b) Effect of transcription inhibition on the MAPK inhibitor-induced increase in HDAC6 protein. H1299 cells were pretreated with $40 \mathrm{nM}$ actinomycin D for $2 \mathrm{~h}$ and were then treated with $20 \mu \mathrm{m}$ PD98059 (PD) for $46 \mathrm{~h}$ prior to western blotting analysis (filled bar). The HDAC6 mRNA level was assessed at $30 \mathrm{~h}$ by qPCR (empty bar). (c) The effects of isoproterenol on C-Raf-MEK-ERK signaling. H1299 cells were treated with isoproterenol for the indicated times, and the phosphorylations of c-Raf (filled bar), MEK (empty bar) and ERK (slant bar) were then analyzed by western blotting and densitometry. $\beta$-Actin was analyzed as a loading control. (d) Effects of ERK activation on the isoproterenol-induced increase in HDAC6 expression. H1299 cells were transfected with constitutively active MEK1 (caMEK1) for $24 \mathrm{~h}$ and were then treated with isoproterenol for $48 \mathrm{~h}$ prior to analysis. Filled bar represents HDAC6 expression and empty bar represents p-ERK. Asterisks $\left(^{*}\right)$ indicate significant differences from the respective control cells $(P<0.05$, Mann-Whitney $U$-test).

H1299 and A549 human lung cancer cells and that ISO treatment caused a decrease in the acetylation of $\alpha$-tubulin and an increase in cell migration in an HDAC6-dependent manner. ISO is an isopropyl analog of epinephrine, which is a major stress hormone, and activates $\beta$-adrenergic receptors, which are the receptors for epinephrine and norepinephrine. Therefore, our findings suggest that stress might increase HDAC6 expression, which might in turn stimulate the migration of cancer cells. Chronic social stress has been found to decrease the expression of HDAC2 in the mouse brain. ${ }^{12}$ However, to the best of our knowledge, this is the first report to reveal that the ISO stress signal increases HDAC6 expression in lung cancer cells.

HDAC6 is predominantly localized in the cytoplasm and regulates many important biological processes, including cell migration, immune synapse formation, transcription, cell proliferation and death, and the degradation of misfolded proteins. ${ }^{17}$ HDAC6 deacetylates $\alpha$-tubulin and thus increases microtubule dynamics to increase cell motility, ${ }^{18}$ and it also modulates actin-dependent cell movement by altering the acetylation status of cortactin. ${ }^{19}$ Thus, we suggest that the increase in HDAC6 expression by ISO stimulate the migration 

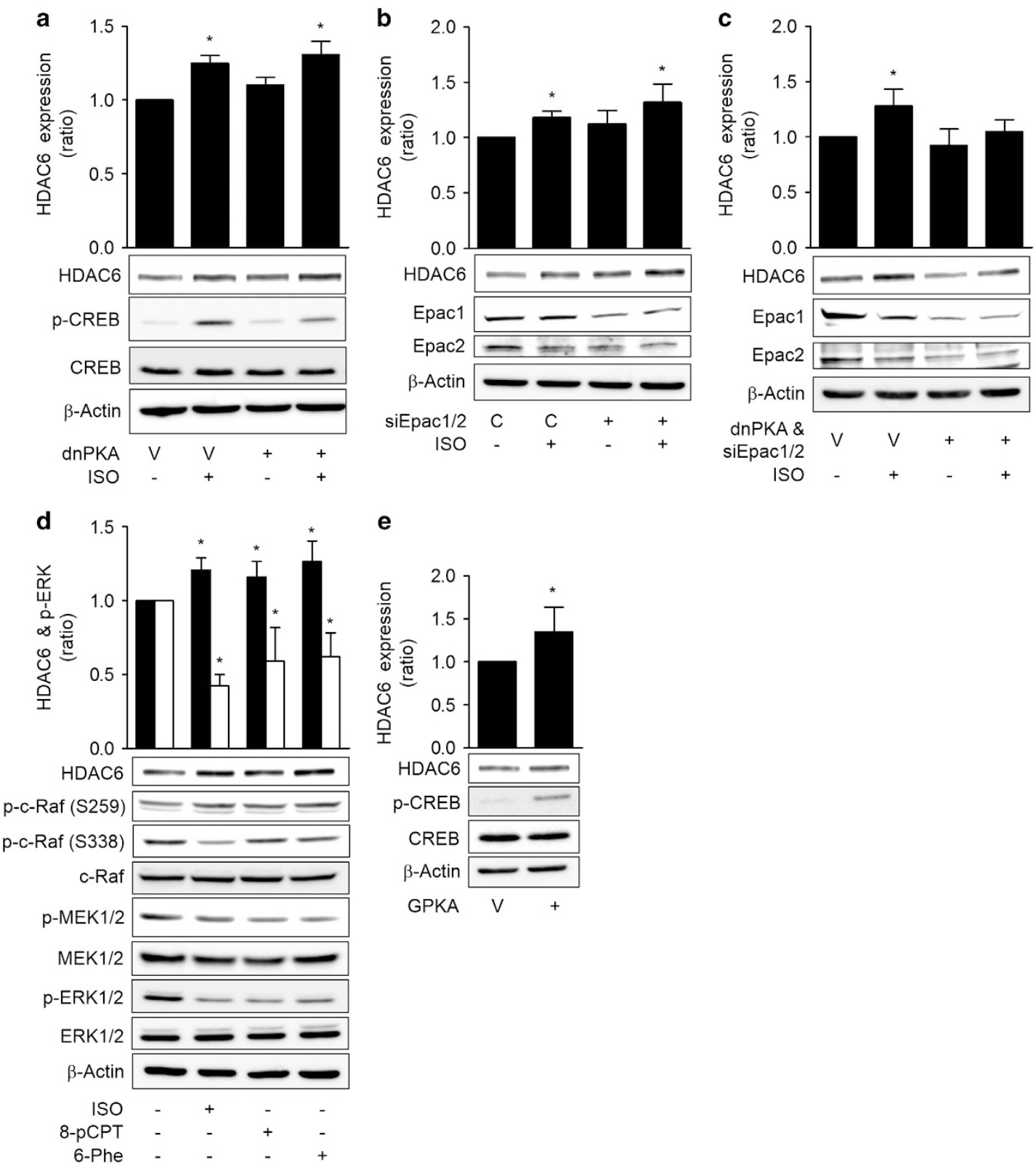

e

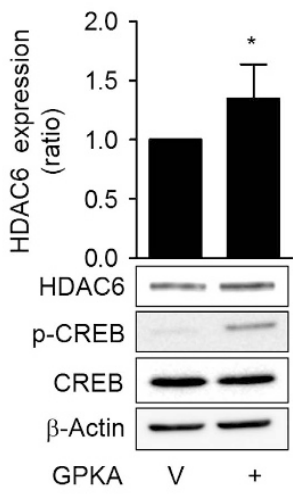

Figure 4 Isoproterenol inhibits c-Raf via the PKA and Epac pathways. (a) Effects of PKA inhibition on the isoproterenol-induced increase in HDAC6 expression. (b) Effects of Epac knockdown on the isoproterenol-induced increase in HDAC6 expression. (c) Effect of simultaneous PKA inhibition and Epac knockdown on the isoproterenol-induced increase in HDAC6 expression. (d) Effects of Epac- and PKA-selective agonists on HDAC6 expression and the c-Raf-MEK-ERK pathways. (e) Effects of PKA activation on HDAC6 expression. H1299 cells were transfected with dominant-negative PKA (dnPKA), Epac1 siRNA, Epac2 siRNA or scrambled control siRNA, incubated for $24 \mathrm{~h}$ and then treated with $20 \mu \mathrm{m}$ isoproterenol (ISO) for $48 \mathrm{~h}$ prior to western blotting analysis. The H1299 cells were also treated for $48 \mathrm{~h}$ with $20 \mu \mathrm{m}$ ISO, $20 \mu \mathrm{m}$ 8-pCPT-2'-0-Me-cAMP (8-pCPT) or $30 \mu \mathrm{m}$ N6-phenyl-cAMP (6-Phe) or transfected with the catalytic subunit of PKA (GPKA) or EGFP vector for $48 \mathrm{~h}$. Then HDAC6 expression was analyzed by western blotting. Filled bar represents HDAC6 expression and empty bar represents p-ERK. Asterisks $(*)$ indicate significant differences from the respective control cells $(P<0.05$, Mann-Whitney U-test).

of lung cancer cells via the deacetylations of $\alpha$-tubulin and cortactin. A similar role of HDAC6 on cell migration and invasion has been reported in hepatocellular carcinomas. ${ }^{20}$ Stress signals, such as norepinephrine, have been reported to enhance invasive potential via the upregulation of matrix metalloproteinases in ovarian and prostate cancer cells. ${ }^{3,21}$ Our paper presents a novel mechanism, that is, the upregulation of HDAC6 expression, by which stress signals might stimulate the invasiveness of cancer cells. In addition, HDAC6 has been reported to deacetylate Hsp90 to regulate glucocorticoid receptor chaperone dynamics in the brain, which provides a promising strategy to reduce the harmful socioaffective effects of stress and glucocorticoids. ${ }^{22,23}$ Furthermore, because HDAC6 are known to interact a variety 


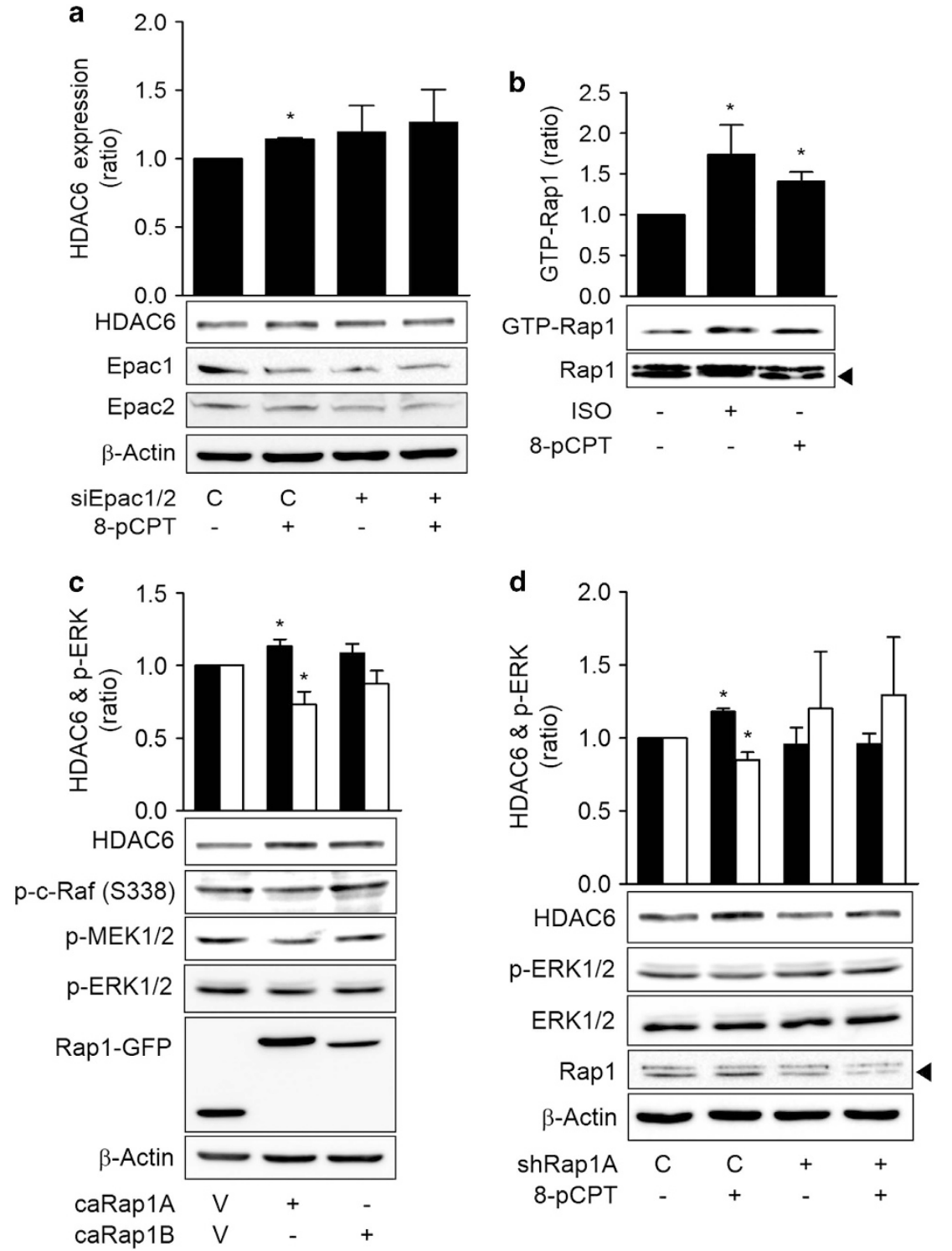

Figure 5 Isoproterenol inhibits c-Raf via the Epac-Rap1 pathways. (a) Effects of Epac knockdown on the 8-pCPT-cAMP-induced increase in HDAC6 expression. Epac1 and Epac2 or control siRNA were transfected for $24 \mathrm{~h}$. Then $20 \mu \mathrm{m}$ 8-pCPT-cAMP treatment was applied for $48 \mathrm{~h}$ prior to western blotting analysis. (b) Effects of isoproterenol on Rap1 activation. $\mathrm{H} 1299$ cells were treated with $20 \mu \mathrm{m}$ ISO or $20 \mu \mathrm{m}$ 8-pCPT-cAMP for 30 min. The GTP-bound Rap1 was then pulled down using the immobilized Rap1-binding domain (RBD) of RalGDS, and western blotting analysis was subsequently performed. (c) Effects of Rap1 activation on ERK phosphorylation (empty bar) and HDA6 expression (filled bar). H1299 cells were transfected with constitutively active Rap1A (caRap1A) and Rap1B (caRap1B) or EGFP control constructs $(\mathrm{V})$ and incubated for $48 \mathrm{~h}$ prior to western blotting analysis. (d) Effects of Rap1A knockdown on 8-pCPT-cAMP-induced ERK phosphorylation and HDAC6 expression. $\mathrm{H} 1299$ cells were transfected with Rap1A shRNA, incubated for $24 \mathrm{~h}$ and then treated with $20 \mu \mathrm{m}$ 8-pCPT-cAMP for $48 \mathrm{~h}$. Asterisks $\left(^{*}\right)$ indicate significant differences from the respective control cells $(P<0.05$, Mann-Whitney $U$-test).

of proteins, ${ }^{24}$ ISO-induced HDAC6 expression levels could regulate the other biological responses involved in cancer progression. HDAC6 can shuttle into the nucleus and deacetylate histone in vitro and in vivo, and therefore the effect of ISO-induced HDAC6 expression on epigenetic regulation need to be further investigated.

We found that ISO increases HDAC6 by activating a cAMP signaling pathway that involves both PKA and Epac, both of which inhibit the c-Raf-MEK-ERK pathway in lung cancer cells. This finding is based on the result that HDAC6 expression was increased when cAMP signaling was activated by the expression of constitutively active Gas or treatment with Gas-coupled receptor agonists (that is, $\mathrm{PGE}_{2}$ and ISO) and an adenylyl cyclase activator (forskolin) in the H1299 and A549 lung cancer cells. Similar to epinephrine and norepinephrine, ISO binds $\beta$-adrenergic receptors to trigger the sequential activation of stimulatory $\mathrm{G}$ proteins, adenylyl cyclases, PKA and Epac signaling. ${ }^{25}$ This finding suggests that HDAC6 expression might be increased not only by the stress signal ISO but also by other signals that increase cAMP 

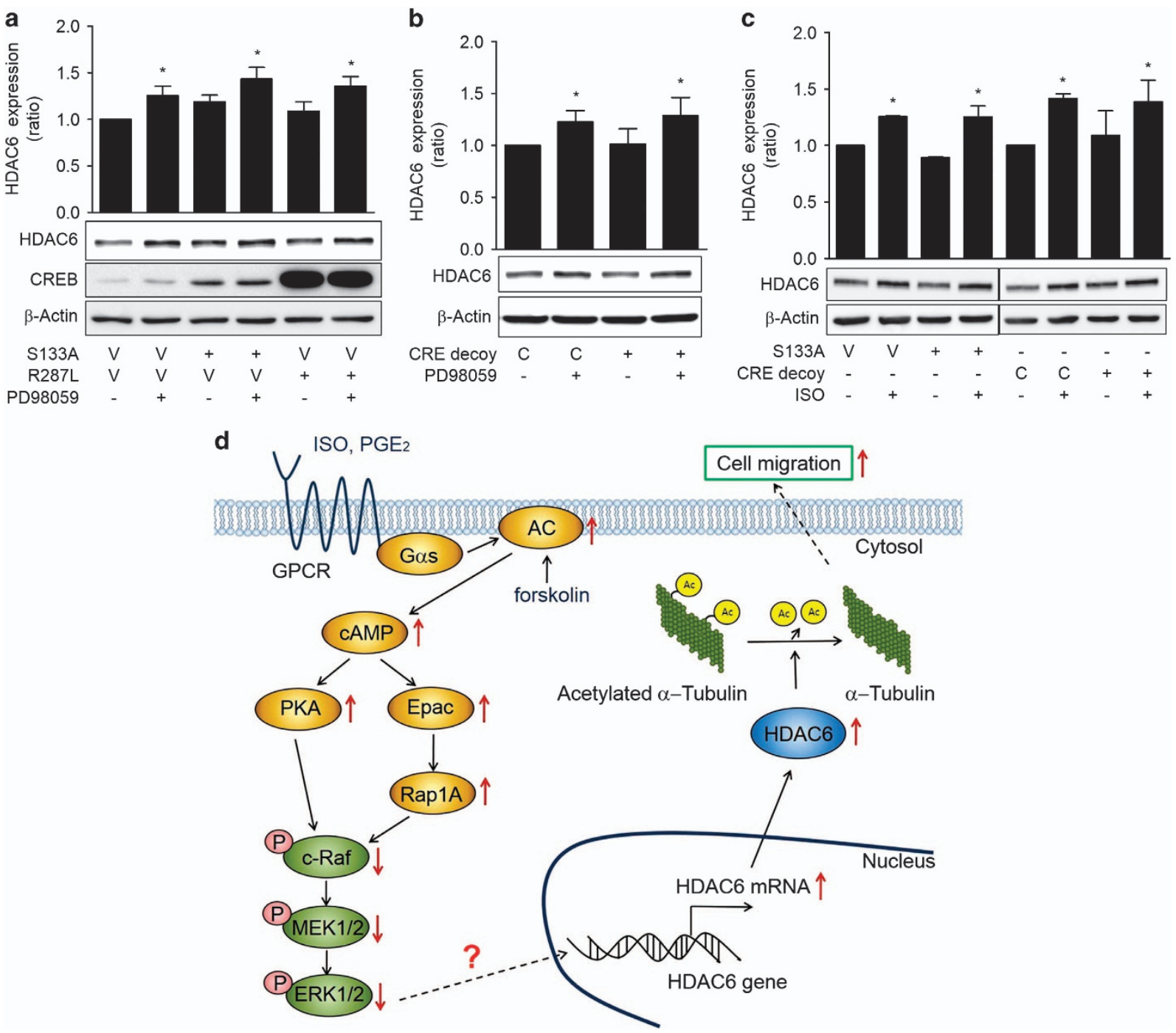

Figure 6 Inhibition of c-Raf-MEK-ERK pathways increases HDAC6 expression in a CREB-independent manner. (a) Effects of dominantnegative CREB on PD98059-induced HDAC6 expression. (b) Effects of CRE decoy oligonucleotides CREB on PD98059-induced HDAC6 expression. (c) Effects of CRE decoy oligonucleotides CREB on isoproterenol-induced HDAC6 expression. H1299 cells were transfected with dominant-negative CREBs (S133A, R287L), respective control vectors (V) or CRE decoy and control (C) oligonucleotides and then incubated for $24 \mathrm{~h}$. The cells were then treated with $20 \mu \mathrm{m}$ PD98059 (PD), isoproterenol (ISO) or dimethyl sulfoxide for $48 \mathrm{~h}$ before western blotting analysis. (d) A suggested mechanism by which isoproterenol increases HDAC6 expression in human lung cancer cells. Isoproterenol increases HDAC6 expression via the PKA- and Epac-mediated inhibitions of c-Raf-MEK-ERK, which result in increased cell migration. The solid lines indicate proven signaling pathways, and the dotted lines indicate potential signaling pathways. GPCR, G-proteincoupled receptor. Asterisks $\left({ }^{*}\right)$ indicate significant differences from the respective control cells $(P<0.05$, Mann-Whitney $U$-test).

concentrations, such as phthalates. $^{26}$ Additionally, cAMP signaling has been reported to stimulate HDAC4 activity in macrophages ${ }^{27}$ and to decrease sirtuin 6 expression in lung cancer cells. ${ }^{28}$ Thus we suggest that stress signals might regulate histone acetylation and gene expression by various ways via activating cAMP signaling.

cAMP signaling regulates various cellular responses by activating three major cAMP effector molecules: PKA, Epac, and cyclic-nucleotide-gated ion channels. ${ }^{29}$ This study revealed that both PKA and Epac mediate the HDAC6-increasing effect of ISO by demonstrating that the inhibition of either PKA or Epac alone did not abolish the effect of ISO on HDAC expression, but the simultaneous inhibition of both PKA and
Epac abolished this effect. Furthermore, treatment with either a PKA- or Epac-selective agonist increased HDAC6 expression. The involvement of PKA was further evidenced by the demonstration that the expression of the PKA catalytic subunit increased HDAC6 expression, and Epac-selective agonist elicited increased HDAC6 expression via a Rap1A-dependent pathway.

In the study on the mechanisms by which PKA and Epac increased HDA6 expression, the PKA and Epac pathways were found to mediate the HADC6-increasing effect of ISO by inhibiting c-Raf-MEK-ERK signaling. This finding is supported by the results that treatment with ISO and selective agonists of PKA or Epac together inhibited c-Raf-MEK-ERK signaling 
pathway, inhibition of the c-Raf-MEK-ERK signaling pathway increased HDAC6 expression and ISO increased HDAC6 expression in an ERK inhibition-dependent manner. ERK is a member of the MAPK family and participates in the regulation of various processes, including cell migration and proliferation and transcription. ERK is activated by following the classical cascade of consecutive activating phosphorylation events: Raf phosphorylates and activates MEK, and activated MEK phosphorylates and activates ERK. ${ }^{30}$ The cAMP and MAPK pathways do not act independently; rather, multiple forms of cross-talk between these pathways can occur. ${ }^{31}$ As shown in our paper, cAMP signaling has previously been reported to inhibit the c-Raf-MEK-ERK pathway by decreasing activating phosphorylation at Ser-338 and increasing inactivating phosphorylation at Ser-259 on the c-Raf protein. ${ }^{32-34}$ The Rap1 protein also inhibits c-Raf activation via the sequestration of c-Raf from Ras through competition for Ras. ${ }^{35,36}$

In the present study, we found that ISO increases HDAC6 expression by inhibiting the c-Raf-MEK-ERK pathway, but the mechanism by which the inhibition of ERK induces HDAC6 expression in a CREB-independent pathway requires elucidation. Phthalates have been reported to increase HDAC6 expression via PKA-dependent CREB phosphorylation, which results in increased CREB binding to a CRE site of the HDAC6 promoter region. ${ }^{26}$ However, the increase in HDAC6 expression induced by ISO or PD98059 was not abolished by blocking the binding of active CREB to CRE sites using dominant-negative CREBs and CRE decoy oligonucleotides in our study, which suggests other mechanisms in ISO-induced increase in HDAC6 expression in lung cancer cells. The ETS transcription factor Erg has been reported to regulate the expression of HDAC6 in human vascular endothelial cells, ${ }^{37}$ and Erg has domains that act as docking platforms for MAPKs; such docking leads to phosphorylation and the enhancement of transactivation activity. ${ }^{38}$ Thus we speculated that ERK signaling might inhibit Erg activation or induce the expression of genes that repress HDAC6 gene transcription.

In conclusion, this study revealed that ISO stress signaling increases HDAC6 expression via a PKA/Epac/ERK-dependent pathway and that the ISO-induced HDAC6 expression increases the migration of lung cancer cells. This study suggests that stress signals might stimulate the migration of cancer cells by increasing the expression of HDAC6 in other cancer cells.

\section{CONFLICT OF INTEREST}

The authors declare no conflict of interest.

\section{ACKNOWLEDGEMENTS}

This work was supported by a grant from the National R\&D Program for Cancer Control, Ministry of Health and Welfare, Republic of Korea (0720540) and by the Basic Science Research Program through the National Research Foundation funded by the Ministry of Education, Science, and Technology (2012R1A1A2044374).
1 Gold PW. The organization of the stress system and its dysregulation in depressive illness. Mol Psychiatry 2015; 20: 32-47.

2 Lillberg K, Verkasalo PK, Kaprio J, Teppo L, Helenius H, Koskenvuo M. Stressful life events and risk of breast cancer in 10,808 women: a cohort study. Am J Epidemiol 2003; 157: 415-423.

3 Sood AK, Bhatty R, Kamat AA, Landen CN, Han L, Thaker PH et al. Stress hormone-mediated invasion of ovarian cancer cells. Clin Cancer Res 2006; 12: 369-375.

4 Wang LP, Jin J, Lv FF, Cao J, Zhang J, Wang BY et al. Norepinephrine attenuates CXCR4 expression and the corresponding invasion of MDAMB-231 breast cancer cells via beta2-adrenergic receptors. Eur Rev Med Pharmacol Sci 2015; 19: 1170-1181.

5 Powell ND, Tarr AJ, Sheridan JF. Psychosocial stress and inflammation in cancer. Brain Behav Immun 2013; 30 Suppl: S41-S47.

6 Archer SY, Hodin RA. Histone acetylation and cancer. Curr Opin Genet Dev 1999; 9: 171-174.

7 Yang XJ, Seto E. HATs and HDACs: from structure, function and regulation to novel strategies for therapy and prevention. Oncogene 2007; 26: 5310-5318.

8 Weichert W, Roske A, Gekeler V, Beckers T, Stephan C, Jung K et al. Histone deacetylases 1,2 and 3 are highly expressed in prostate cancer and HDAC2 expression is associated with shorter PSA relapse time after radical prostatectomy. Br J Cancer 2008; 98: 604-610.

9 Glozak MA, Seto E. Histone deacetylases and cancer. Oncogene 2007; 26: 5420-5432.

10 West AC, Johnstone RW. New and emerging HDAC inhibitors for cancer treatment. J Clin Invest 2014; 124: 30-39.

11 Flaisher-Grinberg S, Persaud SD, Loh HH, Wei LN. Stress-induced epigenetic regulation of kappa-opioid receptor gene involves transcription factor c-Myc. Proc Natl Acad Sci USA 2012; 109: 9167-9172.

12 Covington HE 3rd, Maze I, LaPlant QC, Vialou VF, Ohnishi YN, Berton 0 et al. Antidepressant actions of histone deacetylase inhibitors. J Neurosci 2009; 29: 11451-11460.

13 Wittchen ES, Aghajanian A, Burridge K. Isoform-specific differences between Rap1A and Rap1B GTPases in the formation of endothelial cell junctions. Small GTPases 2011; 2: 65-76.

14 Bok J, Zha XM, Cho YS, Green SH. An extranuclear locus of cAMP-dependent protein kinase action is necessary and sufficient for promotion of spiral ganglion neuronal survival by cAMP. J Neurosci 2003; 23: 777-787.

15 Kim SY, Seo M, Kim Y, Lee YI, Oh JM, Cho EA et al. Stimulatory heterotrimeric GTP-binding protein inhibits hydrogen peroxide-induced apoptosis by repressing BAK induction in SH-SY5Y human neuroblastoma cells. J Biol Chem 2008; 283: 1350-1361.

16 Park SJ, Ahmad F, Philp A, Baar K, Williams T, Luo H et al. Resveratrol ameliorates aging-related metabolic phenotypes by inhibiting CAMP phosphodiesterases. Cell 2012; 148: 421-433.

17 Valenzuela-Fernandez A, Cabrero JR, Serrador JM, Sanchez-Madrid F. HDAC6: a key regulator of cytoskeleton, cell migration and cell-cell interactions. Trends Cell Biol 2008; 18: 291-297.

18 Tran AD, Marmo TP, Salam AA, Che S, Finkelstein E, Kabarriti R et al. HDAC6 deacetylation of tubulin modulates dynamics of cellular adhesions. J Cell Sci 2007; 120: 1469-1479.

19 Zhang X, Yuan Z, Zhang Y, Yong S, Salas-Burgos A, Koomen J et al. HDAC6 modulates cell motility by altering the acetylation level of cortactin. Mol Cell 2007; 27: 197-213.

20 Kanno K, Kanno S, Nitta H, Uesugi N, Sugai T, Masuda T et al. Overexpression of histone deacetylase 6 contributes to accelerated migration and invasion activity of hepatocellular carcinoma cells. Oncol Rep 2012; 28: 867-873.

21 Barbieri A, Bimonte S, Palma G, Luciano A, Rea D, Giudice A et al. The stress hormone norepinephrine increases migration of prostate cancer cells in vitro and in vivo. Int $\mathrm{J}$ Oncol 2015; 47: 527-534.

22 Espallergues J, Teegarden SL, Veerakumar A, Boulden J, Challis C, Jochems $\mathrm{J}$ et al. HDAC6 regulates glucocorticoid receptor signaling in serotonin pathways with critical impact on stress resilience. J Neurosci 2012; 32: 4400-4416.

23 Jochems J, Teegarden SL, Chen Y, Boulden J, Challis C, Ben-Dor GA et al. Enhancement of stress resilience through histone deacetylase 6-mediated regulation of glucocorticoid receptor chaperone dynamics. Biol Psychiatry 2015; 77: 345-355.

$24 \mathrm{Li} \mathrm{Y,} \mathrm{Shin} \mathrm{D,} \mathrm{Kwon} \mathrm{SH.} \mathrm{Histone} \mathrm{deacetylase} 6$ plays a role as a distinct regulator of diverse cellular processes. FEBS J 2013; 280: 775-793. 
25 Schuller HM. Effects of tobacco constituents and psychological stress on the beta-adrenergic regulation of non-small cell lung cancer and pancreatic cancer: implications for intervention. Cancer Biomark 2013; 13: 133-144.

26 Hsieh TH, Tsai CF, Hsu CY, Kuo PL, Lee JN, Chai CY et al. Phthalates induce proliferation and invasiveness of estrogen receptor-negative breast cancer through the AhR/HDAC6/c-Myc signaling pathway. FASEB J 2012; 26: 778-787.

27 Luan B, Goodarzi MO, Phillips NG, Guo X, Chen YD, Yao J et al. Leptin-mediated increases in catecholamine signaling reduce adipose tissue inflammation via activation of macrophage HDAC4. Cell Metab 2014; 19: 1058-1065.

28 Kim EJ, Juhnn YS. Cyclic AMP signaling reduces sirtuin 6 expression in non-small cell lung cancer cells by promoting ubiquitin-proteasomal degradation via inhibition of the Raf-MEK-ERK (Raf/mitogen-activated extracellular signal-regulated kinase/extracellular signal-regulated kinase) pathway. J Biol Chem 2015; 290: 9604-9613.

29 Lefkimmiatis K, Zaccolo M. cAMP signaling in subcellular compartments. Pharmacol Ther 2014; 143: 295-304.

30 Kyriakis JM, Avruch J. Mammalian MAPK signal transduction pathways activated by stress and inflammation: a 10-year update. Physiol Rev 2012; 92: 689-737.

31 Gerits N, Kostenko S, Shiryaev A, Johannessen M, Moens U. Relations between the mitogen-activated protein kinase and the cAMP-dependent protein kinase pathways: comradeship and hostility. Cell Signal 2008; 20: 1592-1607.

32 Mason CS, Springer CJ, Cooper RG, Superti-Furga G, Marshall CJ, Marais R. Serine and tyrosine phosphorylations cooperate in Raf-1, but not B-Raf activation. EMBO J 1999; 18: 2137-2148.

33 Dumaz N, Marais R. Protein kinase A blocks Raf-1 activity by stimulating 14-3-3 binding and blocking Raf-1 interaction with Ras. J Biol Chem 2003; 278: 29819-29823.
34 Li Y, Takahashi M, Stork PJ. Ras-mutant cancer cells display B-Raf binding to Ras that activates extracellular signal-regulated kinase and is inhibited by protein kinase A phosphorylation. J Biol Chem 2013; 288: 27646-27657.

35 Nikam VS, Wecker G, Schermuly R, Rapp U, Szelepusa K, Seeger W et al. Treprostinil inhibits the adhesion and differentiation of fibrocytes via the cyclic adenosine monophosphate-dependent and Ras-proximate proteindependent inactivation of extracellular regulated kinase. Am J Respir Cell Mol Biol 2011; 45: 692-703.

36 Stork PJ. Does Rap1 deserve a bad Rap? Trends Biochem Sci 2003; 28 267-275.

37 Birdsey GM, Dryden NH, Shah AV, Hannah R, Hall MD, Haskard DO et al. The transcription factor Erg regulates expression of histone deacetylase 6 and multiple pathways involved in endothelial cell migration and angiogenesis. Blood 2012; 119: 894-903.

38 Adamo P, Ladomery MR. The oncogene ERG: a key factor in prostate cancer. Oncogene (e-pub ahead of print 27 April 2015; doi: 10.1038/onc.2015.109).

(1)(2) This work is licensed under a Creative Commons Attribution-NonCommercial-ShareAlike $\quad 4.0$

International License. The images or other third party material in this article are included in the article's Creative Commons license, unless indicated otherwise in the credit line; if the material is not included under the Creative Commons license, users will need to obtain permission from the license holder to reproduce the material. To view a copy of this license, visit http://creativecommons.org/licenses/by-nc-sa/4.0/

Supplementary Information accompanies the paper on Experimental \& Molecular Medicine website (http://www.nature.com/emm) 